\title{
ON REPRESENTATIONS OF DIALGEBRAS AND CONFORMAL ALGEBRAS
}

\author{
PAVEL KOLESNIKOV
}

\begin{abstract}
In this note, we observe a relation between dialgebras (in particular, Leibniz algebras) and conformal algebras. The purpose is to show how the methods of conformal algebras help solving problems on dialgebras, and, conversely, how the ideas of dialgebras work for conformal algebras.
\end{abstract}

\section{Conformal Algebras}

The notion of a conformal algebra was introduced in [40] (in [29], a similar notion appeared under the name of a vertex Lie algebra). This notion is an important tool for studying vertex operator algebras. The latter came into algebra from mathematical physics (namely, from the 2-dimensional conformal field theory, what explains the name "conformal algebra"), that was initiated by [1. The algebraic essence of vertex operator structures was extracted in [35] and later developed in a series of works, e.g., [14, 10, 13. The relations between vertex and conformal algebras are very much similar to the relations between ordinary associative and Lie algebras.

In conformal field theory, the operator product expansion (OPE) describes the commutator of two fields. Let $V$ be a (complex) space of states, and let $Y: V \rightarrow$ End $V\left[\left[z, z^{-1}\right]\right], Y: b \mapsto Y(b, z)$, be a state-field correspondence of a vertex algebra. Then the commutator of two fields can be expressed as a finite distribution

$$
[Y(a, w), Y(b, z)]=\sum_{n \geq 0} \frac{1}{n !} Y\left(c_{n}, z\right) \frac{\partial^{n} \delta(w-z)}{\partial z^{n}}, \quad a, b \in V,
$$

where $c_{n} \in V, \delta(w-z)=\sum_{m \in \mathbb{Z}} w^{m} z^{-m-1}$ is the formal delta-function. The formal Fourier transformation

$$
\left[Y(a, z)_{\lambda} Y(b, z)\right]=\operatorname{Res}_{w=0} \exp \{\lambda(w-z)\}[Y(a, w), Y(b, z)]
$$

is called the $\lambda$-bracket on the space of fields $\{Y(a, z) \mid a \in V\}$. Here $\lambda$ is a new formal variable, and $\operatorname{Res}_{w=0} F(w, z)$ means the residue at $w=0$, i.e., the formal series in $z$ that is a coefficient of $F(w, z)$ at $w^{-1}$.

The algebraic properties of the $\lambda$-bracket (11) lead to the formal definition of a conformal algebra over a field $\mathbb{k}$ of characteristic 0 .

Definition 1 (40]). A conformal algebra is a left (unital) module $C$ over the polynomial algebra $H=\mathbb{k}[T]$ endowed with a binary $\mathbb{k}$-linear operation

$$
\left(\cdot{ }_{\lambda} \cdot\right): C \otimes C \rightarrow C[\lambda]
$$

Key words and phrases. Leibniz algebra; Dialgebra; Vertex operator algebra; Conformal algebra. 
such that $\left(T a_{\lambda} b\right)=-\lambda\left(a_{\lambda} b\right),\left(a_{\lambda} D b\right)=(T+\lambda)\left(a_{\lambda} b\right)$.

In terms of fields, $T$ is just the ordinary derivation with respect to $z$.

Every conformal algebra can be represented by formal distributions over an ordinary algebra. Let $C$ be an object described by Definition 11. Consider the space of Laurent polynomials $\mathbb{k}\left[t, t^{-1}\right]$ as a right $H$-module with respect to the following action: $f(t) T=-f^{\prime}(t)$. Then

$$
\mathcal{A}(C)=\mathbb{k}\left[t, t^{-1}\right] \otimes_{H} C
$$

carries the natural algebra structure:

$$
\left(f \otimes_{H} a\right) \cdot\left(g \otimes_{H} b\right)=\left(g \otimes_{H} 1\right)\left(f \otimes_{H}\left(a_{-T} b\right)\right), \quad a, b \in C, f, g \in \mathbb{k}\left[t, t^{-1}\right] .
$$

The space of formal distributions $\mathcal{A}(C)\left[\left[z, z^{-1}\right]\right]$ that consists of all series

$$
Y(a, z)=\sum_{n \in \mathbb{Z}}\left(t^{n} \otimes_{H} a\right) z^{-n-1}, \quad a \in C,
$$

can be endowed with the action of $T=d / d z$ and with a $\lambda$-bracket $\left(\cdot{ }_{\lambda} \cdot\right)$ similar to (1), where the commutator is replaced with the ordinary product of distributions. Then

$$
\left(Y(a, z)_{\lambda} Y(b, z)\right)=Y\left(\left(a_{\lambda} b\right), z\right), \quad a, b \in C,
$$

i.e., $C$ is isomorphic to a formal distribution conformal algebra over $\mathcal{A}(C)$.

The algebra $\mathcal{A}(C)$ is called the coefficient algebra [30] of $C$, or annihilation algebra [40].

Definition 2 ([30]). Let $\mathcal{V}$ be a variety of algebras (associative, alternative, Lie, etc.). Then a conformal algebra $C$ is said to be $\mathcal{V}$-conformal algebra if $\mathcal{A}(C)$ belongs to $\mathcal{V}$.

Associative and Lie conformal algebras, their representations, and cohomologies have been studied in a series of papers, e.g., 6, 2, 37, 31, 9, 4, 41. In particular, associative conformal algebras naturally appear in the study of representations of Lie conformal algebras.

Example 1. Consider one of the simplest (though important) examples of conformal algebras. Suppose A is an ordinary algebra (not necessarily associative or Lie). Then the free $H$-module

$$
\operatorname{Cur} A=H \otimes A
$$

generated by the space $A$ endowed with the $\lambda$-bracket $(f(T) \otimes a)_{\lambda}(g(T) \otimes b)=$ $f(-\lambda) g(T+\lambda) \otimes a b$, is called the current conformal algebra.

If $A$ belongs to a variety $\mathcal{V}$ defined by a family of polylinear identities then Cur $A$ is a $\mathcal{V}$-conformal algebra.

Certainly, current conformal algebras and their subalgebras do not exhaust the entire class of conformal algebras. For example, $W=\mathbb{k}[T, x]$ with respect to the operation

$$
\left(f(T, x)_{\lambda} g(T, x)\right)=f(-\lambda, T) g(T+\lambda, x+\lambda)
$$

is an associative conformal algebra (called Weyl conformal algebra [30]), and Vir $=$ $\mathbb{k}[T]$ with respect to

$$
\left(f(T)_{\lambda} g(T)\right)=f(-\lambda) g(T+\lambda)(T+2 \lambda)
$$

is a Lie conformal algebra (called Virasoro conformal algebra [40]).

Conformal algebra is said to be finite if it is a finitely generated $H$-module. 


\section{Dialgebras}

The following notion appears naturally from a certain noncommutative analogue of Lie homology theory.

Definition 3 ([15]). A (left) Leibniz algebra is a linear space $L$ with a bilinear operation $[\cdot, \cdot]$ such that

$$
[x,[y, z]]=[[x, y], z]+[y,[x, z]], \quad x, y, z \in L .
$$

The defining identity means that the operator of left multiplication $[x, \cdot]$ is a derivation of $L$. Leibniz algebras are the most popular noncommutative generalizations of Lie algebras. The following structures play the role of associative enveloping algebras for Leibniz algebras.

Definition 4 (16]). An associative dialgebra (or diassociative algebra) is a linear space $D$ endowed with two bilinear operations $(\cdot \dashv \cdot),(\cdot \vdash \cdot)$ such that

$$
\begin{aligned}
x \dashv(y \vdash z)= & x \dashv(y \dashv z), \quad(x \dashv y) \vdash z=(x \vdash y) \vdash z, \\
& x \vdash(y \vdash z)=(x \vdash y) \vdash z, \\
& x \dashv(y \dashv z)=(x \dashv y) \dashv z, \\
& x \vdash(y \dashv z)=(x \vdash y) \dashv z,
\end{aligned}
$$

for all $x, y, z \in D$.

In particular, the operation $[a, b]=a \vdash b-b \dashv a, a, b \in D$, turns a diassociative algebra $D$ into a Leibniz algebra denoted by $D^{(-)}$.

A systematical study of diassociative algebras was performed in [25]. Also, in 24] and [1] the notions of alternative and commutative dialgebras were introduced. These definitions also appear in the general categorical approach using the language of operads [21.

Shortly speaking, an operad $A$ is a collection of spaces $A(n), n \geq 1$, such that a composition rule $A(n) \otimes A\left(m_{1}\right) \otimes \cdots \otimes A\left(m_{n}\right) \rightarrow A\left(m_{1}+\cdots+m_{n}\right)$ and an action of a symmetric group are defined in such a way that some natural axioms hold (associativity of a composition, existence of a unit in $A(1)$, and equivariance of the composition with respect to the symmetric group action).

A linear space $A$ over a field $\mathbb{k}$ may be considered as an operad (see, e.g., 23] as a general reference), where $A(n)=\operatorname{Hom}\left(A^{\otimes n}, A\right)$. In the free operad denoted by Alg, the spaces $\operatorname{Alg}(n)$ are spanned by (planar) binary trees with $n$ leaves.

An algebra structure on a linear space $A$ is just a functor of operads Alg $\rightarrow A$. If $\mathcal{V}$ is a variety of algebras defined by polylinear identities then there exists a free $\mathcal{V}$-operad $\mathcal{V}$-Alg built on polylinear polynomials of the free $\mathcal{V}$-algebra. There exists a canonical functor $\mathrm{Alg} \rightarrow \mathcal{V}$-Alg, and it is clear that an algebra $A$ belongs to $\mathcal{V}$ if and only if there exists a functor $\mathcal{V}$-Alg $\rightarrow A$ such that the following diagram is commutative:

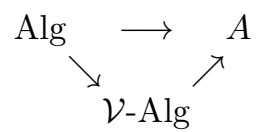

A similar definition works for dialgebras. An operad Dialg whose spaces are spanned by planar binary trees with 2-colored vertices (colors 1 and 2 stand for $\vdash$ and $\dashv$, respectively) has an image equivalent to the Hadamard product $\operatorname{Alg} \otimes \mathcal{E}$, 
where $\mathcal{E}$ is the free $\mathcal{V}_{c}$-operad corresponding to the variety $\mathcal{V}_{c}$ of associative and commutative dialgebras (Perm-algebras), $\operatorname{dim} \mathcal{E}(n)=n$ (see [11, 21] for details).

Suppose $\mathcal{V}$ is a variety of algebras defined by polylinear identities. For a linear space $D$, a functor Dialg $\rightarrow D$ defines two bilinear operations $\vdash$ and $\dashv$ on $D$. Conversely, any system $(D, \vdash, \dashv)$ may be considered as a functor Dialg $\rightarrow D$.

Definition 5 (21]). A linear space $D$ with two bilinear operations $\vdash$ and $\dashv$ is said to be di-V-algebra if there exists a functor $\mathcal{V}$-Alg $\otimes \mathcal{E} \rightarrow D$ such that the following diagram is commutative:

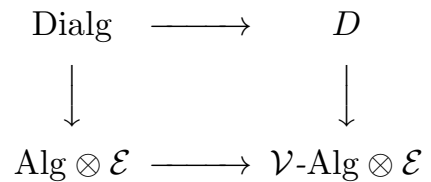

We will mainly use the term "di- $\mathcal{V}$-algebra", but this is the same as " $\mathcal{V}$-dialgebra". The last definition is easy to translate into the language of identities. First, identify $\operatorname{Alg}(n)$ with the space of polylinear non-associative polynomials in $x_{1}, \ldots, x_{n}$; for Dialg we have a similar interpretation. Next, consider the following linear maps $\Psi_{k}: \operatorname{Alg}(n) \rightarrow \operatorname{Dialg}(n), k=1, \ldots, n:$

$$
\Psi_{k}:\left(x_{j_{1}} \ldots x_{k} \ldots x_{j_{n}}\right) \mapsto\left(x_{j_{1}} \vdash \ldots \vdash x_{k} \dashv \ldots \dashv x_{j_{n}}\right),
$$

assuming the bracketing (...) on monomials is preserved. Then we have

Theorem 1 (21]). Assume $\left\{f_{i} \mid i \in I\right\}$ is the family of polylinear defining identities of a variety $\mathcal{V}$. Then $D$ is a di-V-algebra if and only if $D$ satisfies the identities $\Psi_{k}\left(f_{i}\right)=0$ for all $i \in I, k=1, \ldots, \operatorname{deg} f_{i}$.

If $\mathcal{V}$ is the variety of Lie algebras then $f=x_{1} x_{2}+x_{2} x_{1}$ is one of its defining identities. Since $\Psi_{1}(f)=x_{1} \dashv x_{2}+x_{2} \vdash x_{1}$, we can describe Lie dialgebras in terms of single operation, say, $[a, b]=a \vdash b$. Then the class of Lie dialgebras coincides with the class of Leibniz algebras.

Note that all di- $\mathcal{V}$-algebras satisfy the relations (3), called 0 -identities [21. The following approach to the definition of varieties of dialgebras was proposed in 34 .

Let $D$ be a dialgebra that satisfies 0 -identities. Then the linear span $D_{0}$ of all elements $a \vdash b-a \dashv b, a, b \in D$, is an ideal of $D$. The quotient $\bar{D}=D / D_{0}$ is an ordinary algebra with a single operation. Moreover, the following actions are well-defined:

$$
\begin{array}{ll}
\bar{D} \otimes D \rightarrow D, & D \otimes \bar{D} \rightarrow D, \\
\left(a+D_{0}\right) \otimes b \mapsto a \vdash b, & a \otimes\left(b+D_{0}\right) \mapsto a \dashv b .
\end{array}
$$

Denote by $\hat{D}$ the split null extension $\bar{D} \oplus D$, assuming $D^{2}=0$.

Theorem 2 (34]). Suppose $\mathcal{V}$ is a variety of algebras with polylinear defining identities. Then $D$ is a di-V-algebra if and only if $D$ satisfies the 0 -identities and $\hat{D}$ is an algebra from $\mathcal{V}$.

A curious relation between conformal algebras and dialgebras was noted in [21]. It turns out that if $C$ is a $\mathcal{V}$-conformal algebra in the sense of Definition 2 then the same linear space endowed with just two operations

$$
a \vdash b=\left.\left(a_{\lambda} b\right)\right|_{\lambda=0}, \quad a \dashv b=\left.\left(a_{\lambda} b\right)\right|_{\lambda=-T}, \quad a, b \in C,
$$


is a di- $\mathcal{V}$-algebra denoted by $C^{(0)}$. Conversely, every di- $\mathcal{V}$-algebra can be embedded into an appropriate $\mathcal{V}$-conformal algebra. The last statement easily follows from Theorem 2 and

Theorem 3 (c.f. 39]). Let $D$ be a dialgebra satisfying the 0-identities. Then the map $D \rightarrow H \otimes \hat{D}, a \mapsto 1 \otimes\left(a+D_{0}\right)+T \otimes a, a \in D$, is an injective homomorphism

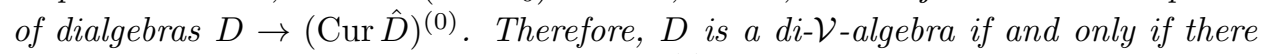
exists a $\mathcal{V}$-algebra $A$ such that $D \subseteq(\operatorname{Cur} A)^{(0)}$.

Thus, there are three equivalent definitions of what is a dialgebra of a given variety provided by Theorems 1, 2, and 3.

\section{Some Classical Theorems for Leibniz Algebras}

Since Leibniz algebras are just Lie dialgebras in the sense of Definition 5, we may use Theorem 3 to get natural generalizations of some classical statements on Lie algebras to the class of Leibniz algebras. These are: the Engel Theorem, the Poincaré - Birkhoff-Witt (PBW) Theorem, and the Ado Theorem.

We will need the following statement (c.f. Theorem 3 in [20]).

Theorem 4. Let $L$ be a Leibniz algebra, and let $V$ be a module over the Lie algebra $\bar{L}$. Then there exists an injective homomorphism $\rho: L \rightarrow(\operatorname{Curgl}(V \oplus(L \otimes V)))^{(0)}$ of Leibniz algebras.

Proof. For every $x \in L$, denote $\bar{x}=x+L_{0} \in \bar{L}$ and define $\rho(x) \in H \otimes \operatorname{gl}(V \oplus(L \otimes V))$ as follows:

$$
\rho(x)=1 \otimes \rho_{0}(x)+T \otimes \rho_{1}(x), \quad \rho_{i}(x) \in \operatorname{gl}(V \oplus(L \otimes V)),
$$

where

$$
\begin{aligned}
& \rho_{0}(x): v \mapsto \bar{x} v, \\
& \rho_{0}(x): a \otimes v \mapsto a \otimes \bar{x} v+[x, a] \otimes v, \\
& \rho_{1}(x): v \mapsto x \otimes v, \\
& \rho_{1}(x): a \otimes v \mapsto 0
\end{aligned}
$$

for all $a \in L, v \in V$. It is clear that $\rho$ is injective $\left(\rho_{1}(x) \neq 0\right.$ for $\left.x \neq 0\right)$. Let us check that $\rho$ is a homomorphism of Leibniz algebras. First, $\left(\rho(x)_{\lambda} \rho(y)\right)=$ $\left[1 \otimes \rho_{0}(x)-1 \otimes \lambda \rho_{1}(x), 1 \otimes \rho_{0}(y)+(T+\lambda) \rho_{1}(y)\right]$. for all $x, y \in L$. Hence, $[\rho(x), \rho(y)]=$ $1 \otimes\left[\rho_{0}(x), \rho_{0}(y)\right]+T \otimes\left[\rho_{0}(x), \rho_{1}(y)\right]$. Next, it is straightforward to compute

$$
\begin{aligned}
& {\left[\rho_{0}(x), \rho_{0}(y)\right]: v+(a \otimes w) \mapsto[\bar{x}, \bar{y}] v+a \otimes[\bar{x}, \bar{y}] w+[[x, y], a] \otimes w,} \\
& {\left[\rho_{0}(x), \rho_{1}(y)\right]: v+(a \otimes w) \mapsto[x, y] \otimes v}
\end{aligned}
$$

for all $v, w \in V, a \in L$. Therefore, $\left[\rho_{0}(x), \rho_{0}(y)\right]=\rho_{0}([x, y]),\left[\rho_{0}(x), \rho_{1}(y)\right]=$ $\rho_{1}([x, y])$, i.e., $[\rho(x), \rho(y)]=\rho([x, y])$.

The following statement immediately follows from Theorem 4 applied to $V=L$.

Theorem 5 ([38, 3, 7]). Let $L$ be a finite-dimensional Leibniz algebra such that all operators $[x, \cdot] \in$ End $L$ are nilpotent. Then $L$ itself is a nilpotent Leibniz algebra.

Recall that for a Lie algebra $L$ the classical PBW Theorem states that the universal enveloping associative algebra $U(L)$ is isomorphic (as a linear space) to the symmetric algebra $S(L)$. For Leibniz algebras, the role of associative envelopes belongs to diassociative algebras. 
Theorem 6 (25, 26]). The universal enveloping diassociative algebra $U d(L)$ of a Leibniz algebra $L$ is isomorphic (as a linear space) to $U(\bar{L}) \otimes L$.

As in the case of Lie algebras, the main technical difficulty in the proof of the PBW Theorem for Leibniz algebras is to show that "normal" monomials are linearly independent. In [22], another proof of this independence was obtained by making use of Gröbner-Shirshov bases theory for diassociative algebras. However, one may just apply Theorem 4 to $V=U(\bar{L})$, see 20 for details.

Another interesting question is similar to the Ado Theorem: Whether a finitedimensional Leibniz algebra can be embedded into a finite-dimensional diassociative algebra? It turns out, the answer is positive. Indeed, it is enough to apply Theorem 4 to $V=\mathbb{k}$, a trivial 1-dimensional module over $\bar{L}$. In particular, we may conclude that an $n$-dimensional Leibniz algebra can be embedded into a diassociative algebra $D$ such that $\operatorname{dim} D \leq 2(n+1)^{2}$.

\section{Di-JORDAN ALGEBRAS}

A diassociative algebra $D$ turns into a Leibniz algebra $D^{(-)}$if we define the bracket $[x, y]=x \vdash y-y \dashv x$. This is natural to expect that if we define new operation

$$
x \circ y=x \vdash y+y \dashv x, \quad x, y \in D,
$$

then the algebra $D^{(+)}=(D, \circ)$ obtained would be a noncommutative analogue of a Jordan algebra. Roughly speaking, it relates to Jordan algebras in the same way as Leibniz algebras relate to Lie algebras.

This is indeed a di-Jordan algebra; the commutativity identity turns into

$$
\Psi_{1}\left(x_{1} x_{2}-x_{2} x_{1}\right)=x_{1} \dashv x_{2}-x_{2} \vdash x_{1},
$$

so we may describe this algebra with only one operation. Objects of this type appeared also in [36, 8].

Definition 6. A di-Jordan algebra is a linear space with a bilinear product satisfying the following identities:

$$
\begin{gathered}
{\left[x_{1}, x_{2}\right] x_{3}=0} \\
\left(x_{1}^{2}, x_{2}, x_{3}\right)=2\left(x_{1}, x_{2}, x_{1} x_{3}\right), \quad x_{1}\left(x_{1}^{2} x_{2}\right)=x_{1}^{2}\left(x_{1} x_{2}\right) .
\end{gathered}
$$

Here $[a, b]$ and $(a, b, c)$ stand for the commutator $a b-b a$ and associator $(a b) c-$ $a(b c)$, respectively. The first identity in (7) comes from the 0 -identities, the second and third appear from the Jordan identity. In 27] these algebras were called semispecial quasi-Jordan algebras.

Recall that a Jordan algebra $J$ is said to be special if there exists an associative algebra $A$ such that $J \subseteq A^{(+)}$. The class of all homomorphic images of all special Jordan algebras is a variety denoted by SJ. This is well-known that SJ does not coincide with the variety of all Jordan algebras. Those defining identities of SJ that do not hold in all Jordan algebras are called special identities (or s-identities, for short).

I was shown in [12] that the minimal degree of an s-identity is equal to 8. However, the description of all s-identities is still an open problem.

For di-Jordan algebras, the same theory makes sense.

Definition 7 (27). A di-Jordan algebra $J$ is said to be special if there exists a diassociative algebra $D$ such that $J \subseteq D^{(+)}$. 
It is clear that the class of all homomorphic images of all special di-Jordan algebras is a variety. Let us denote this variety by DiSJ. The notion of an sidentity for di-Jordan algebras is a natural generalization of s-identities for Jordan algebras.

The following statement was proved in 27] by making use of computer algebra methods.

Theorem 7 ([27]). 1. For di-Jordan algebras, there are no s-identities of degree $\leq 7$

2. There exists an identity of degree 8 that holds on all special di-Jordan algebras and on all Jordan algebras, but does not hold on all di-Jordan algebras.

On the other hand, the variety SJ leads to the notion of a di-SJ-algebra by Definition 5. It turns out that these two different approaches lead to the same class of dialgebras.

Theorem 8 (42]). The variety of di-SJ-algebras coincides with DiSJ.

This fact allows to deduce a correspondence between s-identities for Jordan algebras and dialgebras.

Theorem 9 ([42]). Let $f\left(x_{1}, \ldots, x_{n}\right)$ be a polylinear s-identity for Jordan algebras. Then $\Psi_{k} f, k=1, \ldots, n$, is an s-identity for di-Jordan algebras. Conversely, if $g\left(x_{1}, \ldots, x_{n}\right)$ is an s-identity for di-Jordan algebras then

$$
g\left(x_{1}, \ldots, x_{n}\right)=\sum_{k=1}^{n} g_{k}, \quad g_{k}=\Psi_{k}\left(f_{k}\right)
$$

for some nonassociative polynomials $f_{k}\left(x_{1}, \ldots, x_{n}\right)$, and at least one of $f_{k}$ is an s-identity for Jordan algebras.

Note that Theorem 9 works for polylinear identities only, so it says nothing about the identity from Theorem $7(2)$.

A series of classical results for special Jordan algebras can be transferred to dialgebras. In particular, the Shirshov-Cohn Theorem states that every 2-generated Jordan algebra is special. It turns out that the free 2 -generated di-Jordan algebra is special, but its homomorphic image may not be special [42]. However, Theorem 3 implies that every 1-generated di-Jordan algebra is special.

Another problem on di-Jordan algebras concerns their relation to Leibniz algebras. The classical Tits - Kantor-Koecher construction allows to build a Lie algebra $T(J)$ for a given Jordan algebra $J$ in such a way that structure of $J$ is closely related with the structure of $T(J)$. This is natural to expect [36 that a similar construction for a di-Jordan algebra should lead to Lie dialgebra, i.e., Leibniz algebra.

Conformal algebras allow to solve this problem.

Let $J$ be a di-Jordan algebra, and let $\hat{J}$ stands for the split null extension $\bar{J} \oplus J$ (see Theorem 21). This is a Jordan algebra, and it follows from Theorem 3 that $J \subseteq(\operatorname{Cur} \hat{J})^{(0)}$.

Denote by

$$
T(\hat{J})=\hat{J}^{+} \oplus S(\hat{J}) \oplus \hat{J}^{-}
$$


the Tits-Kantor-Koecher construction [19, 28, 17, for $\hat{J}$. Here $\hat{J}^{ \pm}$are linear spaces isomorphic to $\hat{J}$, and $S(\hat{J}) \subseteq$ End $\hat{J} \oplus \operatorname{Der} \hat{J}$ is spanned by

$$
U_{a, b}=L_{a b}+\left[L_{a}, L_{b}\right], \quad a, b \in \hat{J},
$$

where $L_{x}$ denotes the operator of left multiplication: $L_{x}(y)=x y$. The images of $a \in J$ in the isomorphic copies $J^{ \pm}$are denoted by $a^{ \pm}$. This is a Lie algebra, $J^{+}$and $J^{-}$are its abelian subalgebras, and $\left[a^{-}, b^{+}\right]=L_{a b}+\left[L_{a}, L_{b}\right]$ for $a, b \in J$. Therefore, $\mathcal{L}(J)=(\operatorname{Cur} T(\hat{J}))^{(0)}$ is a Leibniz algebra. Then the elements $1 \otimes\left(a+J_{0}\right)^{ \pm}+T \otimes a^{ \pm} \in$ $\mathcal{L}(J), a \in J$, generate a Leibniz algebra $T(J)$

Theorem 10 ([39]). Let $J$ be a di-Jordan algebra. Then $T(J)$ is a solvable Leibniz algebra if and only if $J$ is a Penico solvable [18]; $T(J)$ is nilpotent if and only if so is $J$.

\section{On Embeddings of Conformal Algebras}

One of the basic facts about associative algebras states that every finite-dimensional associative algebra $A$ can be presented by matrices. Indeed, even if $A$ does not contain a unit element, we may consider $A^{\#}=A \oplus \mathbb{k} 1$, and then there is a faithful representation $L: A \rightarrow$ End $A^{\#}, L(a): x \rightarrow a x$.

For a conformal algebra $C$ of rank $n$ over $H$, the role of End $A$ belongs to Cend $C$, which is isomorphic to the conformal algebra of $n \times n$ matrices over the Weyl conformal algebra. The following properties define an analogue of the unit element for conformal algebras.

Definition 8 (4]). Suppose $C$ is a conformal algebra. An element $e \in C$ is said to be a (conformal) unit in $C$ if $\left.\left(e_{\lambda} x\right)\right|_{\lambda=0}=x$ for all $x \in C$ and $e_{\lambda} e=e$.

Associative conformal algebra with a unit has a very natural structure.

Proposition 1 (4]). Let $C$ be a semisimple associative conformal algebra with a unit. Then there exists an associative algebra $A$ with a locally nilpotent derivation $\partial$ such that $C \simeq H \otimes A$ with respect to the operation

$$
(f(T) \otimes a)_{\lambda}(g(T) \otimes b)=f(-\lambda) g(T+\lambda) \otimes a \exp \{\lambda \partial\} b, \quad a, b \in A, f, g \in H .
$$

Remark 1. In particular, if $\partial=0$ then such $C$ is just the current algebra Cur $A$; if $A=\mathbb{k}[x]$ and $\partial=d / d x$ then $C$ is the Weyl conformal algebra.

This is the reason why the following problem [5] makes sense: Is it possible to join a conformal unit to an associative conformal algebra. Moreover, a finite associative conformal algebra can be embedded into matrices over the conformal Weyl algebra if and only if one can join a unit to this conformal algebra. The following statement answers positively to this question.

Theorem 11 (33). If $C$ is a finite associative conformal algebra which is a torsion-free $H$-module then there exists an associative conformal algebra $C_{e}$ with a unit such that $C \subseteq C_{e}$.

The last Theorem does not hold for all conformal algebras. For example, consider the free $H$-module $C$ generated by the space $W=\mathbb{k}[x] \oplus \mathbb{k} w$ with the following operation on generators:

$$
W_{\lambda} w=0, \quad f(x)_{\lambda} g(x)=f(x-T-\lambda) g(x), \quad w_{\lambda} f(x)=f(T) w,
$$


for $f, g \in \mathbb{k}[x]$. This is an associative conformal algebra. Assume there exists a unital conformal algebra $C_{e}$ with unit $e$ such that $C \subseteq C_{e}$. Then associativity implies

$$
\left(\left(e_{\lambda} w\right)_{0} x^{n}\right)=\left(e_{\lambda}\left(w_{-\lambda} x^{n}\right)\right)=e_{\lambda} T^{n} w=(T+\lambda)^{n}\left(e_{\lambda} w\right), n \geq 1 .
$$

This is impossible since $\left(e_{\lambda} w\right)$ is a polynomial in $\lambda$, and its degree does not depend on the choice of $n$, except for $\left(e_{\lambda} w\right)=0$. But $\left(e_{0} w\right)=w$ by the definition of a conformal unit. The contradiction obtained shows that $C_{e}$ does not exist.

Another problem on embeddings of conformal algebras concerns the following observation. If $C$ is an associative conformal algebra with operations $T: C \rightarrow C$ and $\left({ }_{\lambda} \cdot\right): C \otimes C \rightarrow C[\lambda]$ then the same module over $H=\mathbb{k}[T]$ with respect to the new operation

$$
\left[x_{\lambda} y\right]=\left(x_{\lambda} y\right)-\left.\left(y_{\mu} x\right)\right|_{\mu=-T-\lambda}
$$

is a Lie conformal algebra [40. It is natural to denote this conformal algebra by $C^{(-)}$.

In contrast to the case of ordinary algebras, there exist Lie conformal algebras that can not be embedded into associative conformal algebras [31. However, it is unknown whether the following statement is true.

Conjecture 1. Suppose $L$ is a finite Lie conformal algebra which is a torsion-free $H$-module. Then $L$ can be embedded into an associative conformal algebra with unit.

It was shown in 2 that every such $L$ has a maximal solvable ideal $R$, so $L / R$ is semisimple.

The conjecture obviously holds for semisimple conformal algebras. In [32, it was shown that a nilpotent Lie conformal algebra can be embedded into a nilpotent associative conformal algebra with the same index of nilpotency. This proves Conjecture1 for nilpotent algebras, but it actually holds in much more general class of Lie conformal algebras that includes finite torsion-free solvable algebras.

The idea comes from the construction of a conformal representation for a Leibniz algebra in Theorem 4 .

Let us first consider a Lie conformal algebra of the type $L=\operatorname{Cur} \mathfrak{g}$, where $\mathfrak{g}$ is a Lie algebra, $\operatorname{dim} \mathfrak{g}<\infty$. This is straightforward to check that the embedding built in the proof of Theorem 4 is in fact a homomorphism of conformal algebras. This proves Conjecture 1 without a reference to the classical Ado Theorem.

In the more general case, the Lie Theorem for conformal algebras proved in 2 allows to deduce the following fact.

Theorem 12 (33). Suppose $L$ is a Lie conformal algebra which is a semi-direct product of a current conformal algebra $\operatorname{Cur} \mathfrak{g}(\operatorname{dim} \mathfrak{g}<\infty)$ and a finite torsion-free solvable Lie conformal algebra $R$. Then there exists a finite-dimensional associative algebra $A$ such that $L \subseteq(\operatorname{Cur} A)^{(-)}$.

\section{ACKNOWLEDGMENTS}

This work was partially supported by RFBR 09-01-00157, SSc 3669.2010.1, SB RAS Integration project N 97, Federal Target Grants 02.740.11.0429, 02.740.11.5191, 14.740.11.0346, and by the ADTP Grant 2.1.1.10726. 


\section{REFERENCES}

[1] A. A. Belavin, A. M. Polyakov, A. B. Zamolodchikov. Infinite conformal symmetry in twodimensional quantum field theory. Nucl. Phys. B, 241(2):333-380, 1984.

[2] A. D'Andrea, V.G. Kac. Structure theory of finite conformal algebras. Sel. Math., New Ser., 4(3):377-418, 1998.

[3] A. Patsourakos. On nilpotent properties of Leibniz algebras. Commun. Algebra, 35(12):38283834, 2007.

[4] A. Retakh. Associative conformal algebras of linear growth. J. Algebra, 237(2):769-788, 2001.

[5] A. Retakh. On associative conformal algebras of linear growth. II. J. Algebra, 304(1):543-556, 2006.

[6] B. Bakalov, V.G. Kac, A.A. Voronov. Cohomology of conformal algebras. Commun. Math. Phys., 200(3):561-598, 1999.

[7] D. W. Barnes. On Engel's Theorem for Leibniz algebras., 2010. arXiv: 1012.0608v1.

[8] M. Bremner. On the definition of quasi-jordan algebra. Commun. Algebra, 38(12):4695-4704, 2010.

[9] C. Boyallian, V.G. Kac, J.I. Liberati. On the classification of subalgebras of Cend_ $N$ and gc_N.J. Algebra, 260(1):32-63, 2003.

[10] C. Dong, J. Lepowsky. Generalized vertex algebras and relative vertex operators. Basel: Birkhäuser, 1993.

[11] F. Chapoton. An endofunctor in the category of operads. (Un endofoncteur de la catégorie des opérades.). In J.-L. Loday et al., editor, Dialgebras and Related Operads., pages 105-110. Berlin: Springer, 2001.

[12] C.M. Glennie. Identities in Jordan algebras. In J. Leech, editor, Computational Problems in Abstract Algebra. Proc. Conf., Oxford, 1967, pages 307-313. Oxford: Pergamon, 1970.

[13] H.-S. Li. Local systems of vertex operators, vertex superalgebras and modules. J. Pure Appl. Algebra, 109(2):143-195, 1996.

[14] I. Frenkel, J. Lepowsky, A. Meurman. Vertex operator algebras and the monster. Boston etc.: Academic Press, Inc., 1988.

[15] J.-L. Loday. A noncommutative version of Lie algebras: Leibniz algebras. (Une version non commutative des algèbres de Lie: Les algèbres de Leibniz.). Enseign. Math., II. Sér., 39(34):269-293, 1993.

[16] J.-L. Loday. Algebras with two associative operations (dialgebras). (Algèbres ayant deux opérations associatives (digèbres).). C. R. Acad. Sci., Paris, Sér. I, 321(2):141-146, 1995.

[17] J. Tits. Une classe d'algèbres de Lie en rélation avec les algèbres de Jordan. Nederl. Akad. Wet., Proc., Ser. A, 65:530-535, 1962.

[18] N. Jacobson. Structure and representations of Jordan algebras. Providence, RI: American Mathematical Society, 1968.

[19] I.L. Kantor. Classification of irreducible transitively differential groups. Sov. Math., Dokl., 5:1404-1407, 1964.

[20] P.S. Kolesnikov. Conformal representations of Leibniz algebras. Sib. Mat. Zh., 49(3):540-547, 2008.

[21] P.S. Kolesnikov. Varieties of dialgebras and conformal algebras. Sib. Mat. Zh., 49(2):322-339, 2008.

[22] L.A. Bokut, Y. Chen, C. Liu. Gröbner-Shirshov bases for dialgebras. Int. J. Algebra Comput., 20(3):391-415, 2010.

[23] T. Leinster. Higher operads, higher categories. Cambridge: Cambridge University Press, 2004.

[24] D. Liu. Steinberg-Leibniz algebras and superalgebras. J. Algebra, 283(1):199-221, 2005.

[25] J.-L. Loday. Dialgebras. In J.-L. Loday et al., editor, Dialgebras and Related Operads., pages 7-66. Berlin: Springer, 2001.

[26] M. Aymon, P.-P. Grivel. Un théorème de Poincaré-Birkhoff-Witt pour les algèbres de Leibniz. (A Poincaré-Birkhoff-Witt theorem for Leibniz algebras). Commun. Algebra, 31(2):527-544, 2003.

[27] M. Bremner, L.A. Peresi. Special identities for quasi-Jordan algebras. Commun. Algebra, 39(7):2313-2337, 2011.

[28] M. Koecher. Imbedding of Jordan algebras into Lie algebras. I. Am. J. Math., 89:787-816, 1967. 
[29] M. Primc. Vertex algebras generated by Lie algebras. J. Pure Appl. Algebra, 135(3):253-293, 1999.

[30] M. Roitman. On free conformal and vertex algebras. J. Algebra, 217(2):496-527, 1999.

[31] M. Roitman. Universal enveloping conformal algebras. Sel. Math., New Ser., 6(3):319-345, 2000.

[32] M. Roitman. On embedding of Lie conformal algebras into associative conformal algebras. $J$. Lie Theory, 15(2):575-588, 2005.

[33] P. Kolesnikov. On finite representations of conformal algebras. J. Algebra, 331(2):169-193, 2011.

[34] A.P. Pozhidaev. 0-dialgebras with bar-unity, Rota-Baxter and 3-Leibniz algebras. In A. Giambruno et al., editor, Groups, Rings and Group Rings., pages 245-256. Providence, RI: American Mathematical Society (AMS), 2009.

[35] R. E. Borcherds. Vertex algebras, Kac-Moody algebras, and the monster. Proc. Natl. Acad. Sci. USA, 84:3068-3071, 1986.

[36] R. Velásquez, R. Felipe. Quasi-Jordan algebras. Commun. Algebra, 36(4):1580-1602, 2008.

[37] S.-J. Cheng, V.G. Kac. Conformal modules. Asian J. Math., 1(1):181-193, 1997.

[38] Sh.A. Ayupov, B.A. Omirov. On Leibniz algebras. In Y. Khakimdjanov et al., editor, Algebra and operators theory, pages 1-13. Dordrecht: Kluwer Academic Publishers, 1998.

[39] V. Gubarev, P. Kolesnikov. The Tits-Kantor-Koecher construction for Jordan dialgebras. Comm. Algebra, 39(2):497-520, 2011.

[40] V. Kac. Vertex algebras for beginners. 2nd ed. Providence, RI: American Mathematical Society, 1998.

[41] V.G. Kac, A. Retakh. Simple Jordan conformal superalgebras. J. Algebra Appl., 7(4):517-533, 2008.

[42] V.Yu. Voronin. Special and exceptional Jordan dialgebras, 2010. arXiv:1011.3683.

Sobolev Institute of Mathematics, Novosibirsk, Russia

E-mail address: pavelsk@math.nsc.ru 